\title{
On some equalities for the Weierstrass modular units of level $p$
}

\author{
by \\ Heima Hayashi (Kumamoto)
}

1. Terminology and statement of results. Let $\mathbb{C}, \mathbb{R}, \mathbb{Q}$ and $\mathbb{Z}$ be respectively the fields of complex, real and rational numbers and the ring of rational integers. For each algebraic number field $F$, we denote the ring of integers in $F$ by $\mathfrak{o}_{F}$. For two numbers (or ideals) $A$ and $B$ in some algebraic number field, let the relation $A \sim B$ mean that $A \mathfrak{o}_{F}=B \mathfrak{o}_{F}$ as an ideal in a sufficiently large algebraic number field $F$. By a $\mathbb{C}$-lattice we mean a free $\mathbb{Z}$-module in $\mathbb{C}$ of rank 2 which spans $\mathbb{C}$ over $\mathbb{R}$. In any $\mathbb{C}$-lattice a basis $\left\{\omega_{1}, \omega_{2}\right\}$ can be chosen so that $\operatorname{Im}\left(\omega_{1} / \omega_{2}\right)>0$. Hereafter we denote the $\mathbb{C}$-lattice $\mathbb{Z} \omega_{1}+\mathbb{Z} \omega_{2}$ simply by $\left[\omega_{1}, \omega_{2}\right]$.

Let $\Omega$ be a $\mathbb{C}$-lattice. The Weierstrass $\wp$-function $\wp_{\Omega}(z)$ attached to $\Omega$ is defined by

$$
\wp_{\Omega}(z)=\frac{1}{z^{2}}+\sum_{\omega \in \Omega \backslash\{0\}}\left[\frac{1}{(z-\omega)^{2}}-\frac{1}{\omega^{2}}\right] .
$$

As usual let $g_{2}(\Omega), g_{3}(\Omega)$ and $\Delta(\Omega)$ be the lattice functions respectively defined by

$$
g_{2}(\Omega)=60 \sum_{\omega \in \Omega \backslash\{0\}} \frac{1}{\omega^{4}}, \quad g_{3}(\Omega)=140 \sum_{\omega \in \Omega \backslash\{0\}} \frac{1}{\omega^{6}}
$$

and

$$
\Delta(\Omega)=g_{2}^{3}(\Omega)-27 g_{3}^{2}(\Omega) .
$$

Let $\tau$ be in the complex upper half plane $\mathfrak{H}$, and let $\Omega_{\tau}=[\tau, 1]$. We write $g_{2}(\tau), g_{3}(\tau)$ and $\Delta(\tau)$ respectively for $g_{2}\left(\Omega_{\tau}\right), g_{3}\left(\Omega_{\tau}\right)$ and $\Delta\left(\Omega_{\tau}\right)$. Let $\Gamma=\mathrm{SL}_{2}(\mathbb{Z})$. For a prime number $p$, let $\Gamma(p)$ and $\Gamma_{0}(p)$ be the subgroups

2000 Mathematics Subject Classification: Primary 11G15, 11G16. 
of $\Gamma$ given by

$$
\begin{aligned}
\Gamma(p) & =\left\{\left(\begin{array}{ll}
a & b \\
c & d
\end{array}\right) \in \Gamma \mid a \equiv d \equiv 1, b \equiv c \equiv 0(\bmod p)\right\}, \\
\Gamma_{0}(p) & =\left\{\left(\begin{array}{ll}
a & b \\
c & d
\end{array}\right) \in \Gamma \mid c \equiv 0(\bmod p)\right\} .
\end{aligned}
$$

As is well known, $\left[\Gamma_{0}(p): \Gamma(p)\right]=p(p-1)$ (cf. [1], [7]). More precisely the $\operatorname{map} \Gamma_{0}(p) \rightarrow(\mathbb{Z} / p \mathbb{Z})^{\times} \times(\mathbb{Z} / p \mathbb{Z})$ given by

$$
\left(\begin{array}{ll}
a & b \\
c & d
\end{array}\right) \mapsto(a, b)(\bmod p)
$$

induces an injective map from the factor group $\Gamma(p) \backslash \Gamma_{0}(p)$ onto $(\mathbb{Z} / p \mathbb{Z})^{\times} \times$ $(\mathbb{Z} / p \mathbb{Z})$.

We define a function $\lambda_{p}$ on $\mathfrak{H}$ by

$$
\lambda_{p}(\tau):=\frac{\wp \Omega_{\tau}\left(\frac{1}{p}\right)-\wp \Omega_{\tau}\left(\frac{\tau+1}{p}\right)}{\wp \Omega_{\tau}\left(\frac{\tau}{p}\right)-\wp \Omega_{\tau}\left(\frac{\tau+1}{p}\right)} .
$$

It is called a Weierstrass modular unit (cf. Kubert-Lang [8]). Especially $\lambda_{2}$ is well known as a function which appears in the Legendre model of elliptic curves (cf. [4], [5]). By the properties of the $\wp$-function, we see that $\lambda_{p}$ is a modular function for $\Gamma(p)$ which is holomorphic and non-zero on $\mathfrak{H}$. Hereafter, when the subscript $\Omega_{\tau}$ is clear from the context, we often write $\wp(z)$ in place of $\wp \Omega_{\tau}(z)$, that is,

$$
\lambda_{p}(\tau)=\frac{\wp\left(\frac{1}{p}\right)-\wp\left(\frac{\tau+1}{p}\right)}{\wp\left(\frac{\tau}{p}\right)-\wp\left(\frac{\tau+1}{p}\right)} .
$$

For $\sigma=\left(\begin{array}{ll}a & b \\ c & d\end{array}\right)$ in $\Gamma_{0}(p)$, we have

$$
\lambda_{p}(\sigma(\tau))=\frac{\wp\left(\frac{d}{p}\right)-\wp\left(\frac{a \tau+b+d}{p}\right)}{\wp\left(\frac{a \tau+b}{p}\right)-\wp\left(\frac{a \tau+b+d}{p}\right)} \quad \text { with } \quad \sigma(\tau)=\frac{a \tau+b}{c \tau+d} .
$$


We consider the function on $\mathfrak{H}$ defined by

$$
\begin{aligned}
\Lambda_{p}(\tau) & :=\prod_{\substack{\sigma \bmod \Gamma(p) \\
\sigma \in \Gamma_{0}(p)}} \lambda_{p}(\sigma(\tau)) \\
& =\prod_{a=1}^{p-1} \prod_{b=0}^{p-1} \frac{\wp\left(\frac{d}{p}\right)-\wp\left(\frac{a \tau+b+d}{p}\right)}{\wp\left(\frac{a \tau+b}{p}\right)-\wp\left(\frac{a \tau+b+d}{p}\right)} .
\end{aligned}
$$

In each factor of the expression (1.2), $d$ is determined modulo $p$ so that $a d \equiv 1(\bmod p)$. It is easy to see that $\Lambda_{p}(\tau)$ is a modular function for $\Gamma_{0}(p)$ which is holomorphic and non-zero on $\mathfrak{H}$.

For example, for $p=2$,

$$
\begin{aligned}
\Lambda_{2}(\tau) & =\frac{\wp\left(\frac{1}{2}\right)-\wp\left(\frac{\tau+1}{2}\right)}{\wp\left(\frac{\tau}{2}\right)-\wp\left(\frac{\tau+1}{2}\right)} \cdot \frac{\wp\left(\frac{1}{2}\right)-\wp\left(\frac{\tau}{2}\right)}{\wp\left(\frac{\tau+1}{2}\right)-\wp\left(\frac{\tau}{2}\right)} \\
& =\lambda_{2}(\tau)\left(1-\lambda_{2}(\tau)\right) .
\end{aligned}
$$

Cougnard gave the following equality:

$$
\Lambda_{2}(\tau) \cdot\left(2^{12} \frac{\Delta(2 \tau)}{\Delta(\tau)}\right)=-2^{4}
$$

([4, Theorem 7]). Also if $p$ is an odd prime number, an equality analogous to (1.3) should be expected. Our first aim is to prove the following

TheORem 1. For any odd prime number $p$,

$$
\Lambda_{p}^{2}(\tau) \cdot\left(p^{12} \frac{\Delta(p \tau)}{\Delta(\tau)}\right)^{(p+1) / 2}=p^{6} .
$$

In particular, if $p \equiv 3(\bmod 4)$, then

$$
\Lambda_{p}(\tau) \cdot\left(p^{12} \frac{\Delta(p \tau)}{\Delta(\tau)}\right)^{(p+1) / 4}=-p^{3} .
$$

Section 2 is devoted to proving Theorem 1. In Section 3 we consider a few applications of Theorem 1 . First we compute the equation relating the function $\Lambda_{p}$ and the modular invariant $j$ (see Proposition 2). Next we apply Theorem 1 to the complex multiplication case. In the expressions (1.1) and (1.2), we can replace $\wp(*)=\wp \Omega_{\tau}(*)$ by the Weber function

$$
h(*):=h_{\Omega_{\tau}}(*):=\frac{-2^{7} \cdot 3^{5} \cdot g_{2}(\tau) \cdot g_{3}(\tau)}{\Delta(\tau)} \wp \Omega_{\tau}(*) .
$$


Namely we may write

$$
\begin{aligned}
\lambda_{p}(\tau):= & \frac{h\left(\frac{1}{p}\right)-h\left(\frac{\tau+1}{p}\right)}{h\left(\frac{\tau}{p}\right)-h\left(\frac{\tau+1}{p}\right)}, \\
\Lambda_{p}(\tau)= & \prod_{a=1}^{p-1} \prod_{b=0}^{p-1} \frac{h\left(\frac{d}{p}\right)-h\left(\frac{a \tau+b+d}{p}\right)}{h\left(\frac{a \tau+b}{p}\right)-h\left(\frac{a \tau+b+d}{p}\right)} .
\end{aligned}
$$

Let $k=\mathbb{Q}(\sqrt{-d})$ with a square free positive integer $d$. For simplicity we assume that $d \neq 1,3$. Let $\tau(\in \mathfrak{H})$ be in $k$ and assume that $\Omega_{\tau}=[\tau, 1]$ is an $\mathfrak{o}_{k}$-ideal. For $(a, b) \in \mathbb{Z} \times \mathbb{Z}$ such that $(a, b) \not \equiv(0,0)(\bmod p),(a \tau+b) / p$ represents a non-zero $p$-division point of $\mathbb{C} /[\tau, 1]$, and hence by the classical theory of complex multiplication (cf. Cassou-Noguès and Taylor [3], Deuring $[6]), h((a \tau+b) / p)$ is an integer belonging to the ray class field $k\left(p \mathfrak{o}_{k}\right)$ over $k$ with conductor $p \mathfrak{o}_{k}$. Therefore $\lambda_{p}(\tau)$ and $\Lambda_{p}(\tau)$ also belong to $k\left(p \mathfrak{o}_{k}\right)$. Using the equality in Theorem 1, we shall show some arithmetic properties of $\Lambda_{p}(\tau)$ (see Theorem 4). In Section 4, we shall treat the arithmetic of $\lambda_{p}(\tau)$. Therein we first show how to compute the equation relating $\lambda_{p}(\tau)$ and the modular invariant $j$, and give a numerical example (Theorem 5). Next we consider the algebraic properties of $\lambda_{p}(\tau)$ in the case of complex multiplication (see Theorem 6).

\section{Proof of Theorem 1. We put}

$$
S=\left(\begin{array}{cc}
0 & -1 \\
1 & 0
\end{array}\right) \quad \text { and } \quad T=\left(\begin{array}{ll}
1 & 1 \\
0 & 1
\end{array}\right) \text {. }
$$

As is well known, $\left[\Gamma: \Gamma_{0}(p)\right]=p+1$, and the following is a complete set of left coset representatives for $\Gamma_{0}(p)$ in $\Gamma$ :

$$
\alpha_{0}=I \quad \text { and } \quad \alpha_{i}=S T^{i-1} \quad(i=1, \ldots, p)
$$

(cf. [1], [7]). Of course $\left\{\alpha_{i}^{-1}\right\}_{0 \leq i \leq p}$ represents all right cosets of $\Gamma / \Gamma_{0}(p)$. The set of cusps of $\Gamma_{0}(p)$ is $\{0, \infty\}$, because $\alpha_{0}(\infty)=\infty$ and $\alpha_{i}(\infty)=0$ $(1 \leq i \leq p)$. Now we know that both $\Lambda_{p}(\tau)$ and $\Delta(p \tau) / \Delta(\tau)$ are modular functions for $\Gamma_{0}(p)$ which are non-zero and holomorphic on $\mathfrak{H}$. We compare their $q$-expansions at the cusps of $\Gamma_{0}(p)$. Using the well known formula

$$
(2 \pi i)^{-12} \Delta(\tau)=q \prod_{n=1}^{\infty}\left(1-q^{n}\right)^{24} \quad \text { with } q=e^{2 \pi i \tau}
$$


(cf. [9]), we have

$$
\frac{\Delta(p \tau)}{\Delta(\tau)}=q^{p-1}\left(1+q R_{0}(q)\right)
$$

where $R_{0}(X)$ is a power series in $X$ with coefficients in $\mathbb{Z}$. On the other hand, by making use of Proposition A-1 in the Appendix, we can describe the $q$-expansion of each factor on the right hand side of (1.2). Moreover, by applying Lemma A-2, we can deduce that

$$
\Lambda_{p}(\tau)=(-1)^{(p-1) / 2} p^{-3 p} q^{-\left(p^{2}-1\right) / 4} \cdot\left(1+q^{1 / p} R_{1}\left(q^{1 / p}\right)\right),
$$

where $R_{1}(X)$ is a power series in $X$ with coefficients in $\mathbb{Z}\left[\zeta_{p}\right]$ and $\zeta_{p}=e^{2 \pi i / p}$. Hence the $q$-expansion of

$$
\Lambda_{p}^{2}(\tau) \cdot\left(p^{12} \frac{\Delta(p \tau)}{\Delta(\tau)}\right)^{(p+1) / 2}
$$

at $\infty$ starts with the constant term $p^{6}$, and this also means that $(2.2)$ is holomorphic at $\infty$. It is also clear that if $p \equiv 3(\bmod 4)$ the leading term of the $q$-expansion of

$$
\Lambda_{p}(\tau) \cdot\left(p^{12} \frac{\Delta(p \tau)}{\Delta(\tau)}\right)^{(p+1) / 4}
$$

at $\infty$ is equal to $-p^{3}$. Next we consider the $q$-expansion at the cusp $0=$ $S(\infty)$. Since

$$
p^{12} \frac{\Delta\left(p S^{-1}(\tau)\right)}{\Delta\left(S^{-1}(\tau)\right)}=p^{12} \frac{\Delta\left(\frac{-p}{\tau}\right)}{\Delta\left(\frac{-1}{\tau}\right)}=\frac{\Delta\left(\frac{\tau}{p}\right)}{\Delta(\tau)}
$$

using (2.1), we see that the leading term of the $q$-expansion of $p^{12} \Delta(p \tau) / \Delta(\tau)$ at 0 is equal to $q^{-(p-1) / p}$. On the other hand, we have

$$
\left.\Lambda_{p}\left(S^{-1}(\tau)\right)=\prod_{a=1}^{p-1} \prod_{b=0}^{p-1} \frac{\wp\left(\frac{d \tau}{p}\right)-\wp\left(\frac{(b+d) \tau-a}{p}\right)}{p}\right)-\wp\left(\frac{(b+d) \tau-a}{p}\right) .
$$

Applying Lemma A-2, we can find the leading term of the $q$-expansion of each factor of (2.3). Then by a tedious check, we see that the $q$-expansion of $\Lambda_{p}\left(S^{-1}(\tau)\right)$ at $\infty$ starts with $q^{\frac{1}{p} \cdot \frac{p^{2}-1}{4}}$, and hence the $q$-expansion of $(2.2)$ at $0=S(\infty)$ starts with the constant term. This means that $(2.2)$ is also holomorphic at 0 . Hence (2.2) is holomorphic on the compact Riemann surface $\Gamma_{0}(p) \backslash \mathfrak{H} \cup\{$ cusps $\}$ and so must be a constant. Moreover since

$$
\lim _{\tau \rightarrow i \infty} \Lambda_{p}^{2}(\tau) \cdot\left(p^{12} \frac{\Delta(p \tau)}{\Delta(\tau)}\right)^{(p+1) / 2}=p^{6}
$$


we have the first equality of Theorem 1 . It is also clear that if $p \equiv 3(\bmod 4)$, then

$$
\Lambda_{p}(\tau) \cdot\left(p^{12} \frac{\Delta(p \tau)}{\Delta(\tau)}\right)^{(p+1) / 4}=-p^{3} .
$$

3. Some arithmetic properties of $\Lambda_{p}(\tau)$. Let $\left\{\alpha_{i}\right\}$ be as in Section 2 . We define

$$
A_{i}(\tau):=\Lambda_{p}\left(\alpha_{i}(\tau)\right) \quad(i=0,1, \ldots, p) .
$$

Then by Theorem 1 , we have

$$
A_{0}^{2}(\tau) \cdot\left(p^{12} \frac{\Delta(p \tau)}{\Delta(\tau)}\right)^{(p+1) / 2}=p^{6}
$$

and for $1 \leq i \leq p$,

$$
A_{i}^{2}(\tau) \cdot\left(\frac{\Delta\left(\frac{\tau+i-1}{p}\right)}{\Delta(\tau)}\right)^{(p+1) / 2}=p^{6} .
$$

As is well known,

$$
\beta_{0}=\left(\begin{array}{cc}
p & 0 \\
0 & 1
\end{array}\right) \quad \text { and } \quad \beta_{i}=\left(\begin{array}{cc}
1 & i-1 \\
0 & p
\end{array}\right) \quad(1 \leq i \leq p)
$$

constitute a complete system of representatives of the left cosets $\Gamma \backslash M_{p}$, where $M_{p}$ is the set of integral matrices of determinant $p$. We define

$$
B_{0}(\tau):=p^{12} \frac{\Delta\left(\beta_{0}(\tau)\right)}{\Delta(\tau)}=p^{12} \frac{\Delta(p \tau)}{\Delta(\tau)}
$$

and

$$
B_{i}(\tau):=\frac{\Delta\left(\beta_{i}(\tau)\right)}{\Delta(\tau)}=\frac{\Delta\left(\frac{\tau+i-1}{p}\right)}{\Delta(\tau)} \quad \text { for } 1 \leq i \leq p .
$$

Then the equalities (3.1) and (3.2) can be restated as follows:

$$
A_{i}^{2}(\tau) \cdot B_{i}(\tau)^{(p+1) / 2}=p^{6} \quad \text { for } i=0,1, \ldots, p .
$$

From the classical theory of complex multiplication (cf. [2], [3], [6]), we know that the polynomial

$$
\Phi_{p}^{(k)}(X):=\prod_{i=0}^{p}\left(X-B_{i}^{k}(\tau)\right)
$$

lies in $\mathbb{Z}[j, X]$, where $j$ is the modular invariant defined by

$$
j(\tau)=\frac{1728 g_{2}^{3}(\tau)}{\Delta(\tau)} .
$$


Hence by using equation (1.3) and Theorem 1, it is possible to give the equation relating the functions $\Lambda_{p}$ and $j$. For example, by numerical computations we obtain the following

Proposition 2. Under the notations as above,

(i) $\Lambda_{2}$ satisfies

or equivalently

$$
\Lambda_{2}^{3}+\frac{1}{2^{8}}(j-768) \Lambda_{2}^{2}+3 \Lambda_{2}-1=0,
$$

$$
j=-2^{8} \frac{\left(\Lambda_{2}-1\right)^{3}}{\Lambda_{2}^{2}} .
$$

(ii) $\Lambda_{3}$ satisfies

$$
\Lambda_{3}^{4}+\frac{1}{3^{9}}\left(j^{2}-1512 j+177876\right) \Lambda_{3}^{3}+\frac{1}{3^{4}}(8 j+2214) \Lambda_{3}^{2}+28 \Lambda_{3}+1=0 .
$$

Remark. The second equality in (i) of Proposition 2 is nothing but the equality given in Lang [9, p. 256].

For any odd prime $p$, we know that

$$
\prod_{i=0}^{p} B_{i}(\tau)=p^{12} \frac{\Delta(p \tau)}{\Delta(\tau)} \prod_{i=1}^{p} \frac{\Delta\left(\frac{\tau+i-1}{p}\right)}{\Delta(\tau)}=p^{12} .
$$

Hence by (3.3), we have

$$
\prod_{i=0}^{p} A_{i}^{2}(\tau)=1
$$

In particular, if $p \equiv 3(\bmod 4)$, we have

$$
\prod_{i=0}^{p} A_{i}(\tau)=1
$$

Hereafter in this section, let $\tau(\in \mathfrak{H})$ be in an imaginary quadratic number field $k(\neq \mathbb{Q}(\sqrt{-1}), \mathbb{Q}(\sqrt{-3}))$, and let $\Omega_{\tau}=[\tau, 1]$ be an $\mathfrak{o}_{k}$-ideal. The following is a fundamental result in the classical theory of complex multiplication (cf. [2], [3], [6], [10]).

Proposition 3. Under the above notations, $B_{i}(\tau)(0 \leq i \leq p)$ are algebraic integers and the following hold:

(i) If $p$ splits in $k$ with $p \mathfrak{o}_{k}=\mathfrak{p} \bar{p}$, then there exists a unique $\beta_{i_{1}}$ (resp. $\beta_{i_{2}}$ ) such that $\beta_{i_{1}}(\tau)$ (resp. $\left.\beta_{i_{2}}(\tau)\right)$ is a basis quotient of $\overline{\mathfrak{p}} \Omega_{\tau}$ (resp. $\left.\mathfrak{p} \Omega_{\tau}\right)$. In this case $B_{i_{1}}(\tau)$ and $B_{i_{2}}(\tau)$ are contained in $\mathcal{H}_{k}$, the Hilbert class field of $k$, and

$$
B_{i_{1}}(\tau) \sim \mathfrak{p}^{12} \quad \text { and } \quad B_{i_{2}}(\tau) \sim \overline{\mathfrak{p}}^{12} .
$$

Moreover, for any $i \neq i_{1}, i_{2}, B_{i}(\tau)$ is a unit. 
(ii) If $p$ is ramified in $k$ with $p \mathfrak{o}_{k}=\mathfrak{p}^{2}$, then there exists a unique $\beta_{i_{1}}$ such that $\beta_{i_{1}}(\tau)$ is a basis quotient of $\mathfrak{p} \Omega_{\tau}$. In this case $B_{i_{1}}(\tau)$ is contained in $\mathcal{H}_{k}$ and

$$
B_{i_{1}}(\tau) \sim p^{6}, \quad B_{i}(\tau) \sim p^{6 / p} \quad \text { for any } i \neq i_{1} .
$$

(iii) If $p$ remains prime in $k$, then $B_{i}(\tau) \sim p^{12 /(p+1)}$ for any $i$.

Combining Theorem 1 and Proposition 3, we have the following

TheOREM 4. Let the notations be as above. Then $\Lambda_{p}(\tau)$ is an algebraic number which is a unit outside the prime divisors of $p$. In particular, $\Lambda_{p}(\tau)$ is a unit if $p$ remains prime in $k$.

4. Some arithmetic properties of $\lambda_{p}(\tau)$. As is well known, $[\Gamma$ : $\Gamma(p)]=p\left(p^{2}-1\right)$ (cf. [1], [7]). Let $\left\{\gamma_{i}\right\}$ be a complete set of left coset representatives for $\pm \Gamma(p)$ in $\Gamma$. We consider the polynomial $G_{p}$ given by

$$
\begin{aligned}
G_{p}(X) & :=\prod_{i=0}^{m-1}\left(X-\lambda_{p}\left(\gamma_{i}(\tau)\right)\right) \\
& =X^{m}+C_{m-1}(\tau) X^{m-1}+\cdots+C_{1}(\tau) X+C_{0}(\tau),
\end{aligned}
$$

where $m=\frac{1}{2} p\left(p^{2}-1\right)$. It is easy to verify that all coefficients $C_{i}(\tau)$ of $G_{p}(X)$ are modular functions for $\Gamma$ and holomorphic on $\mathfrak{H}$. Moreover by applying Proposition A-1 and Lemma A-2 of the Appendix, we can verify that the $q$-expansions of $C_{i}(\tau)$ all lie in $\mathbb{Z}\left[1 / p, \zeta_{p}\right]((q))$, the ring of formal Laurent series in $q$ with coefficients in $\mathbb{Z}\left[1 / p, \zeta_{p}\right]$, where $\zeta_{p}=e^{2 \pi i / p}$. Then from the $q$-expansion principle (cf. $[3, \mathrm{Ch} .7]$ ), we can deduce that $C_{i}(\tau)$ are all contained in $\mathbb{Z}\left[1 / p, \zeta_{p}\right][j]$, the ring of polynomials in $j$ with coefficients in $\mathbb{Z}\left[1 / p, \zeta_{p}\right]$.

To get an explicit expression of $C_{i}(\tau)$ as a polynomial in $j$, for example, we only have to interpolate the $q$-expansion of $C_{i}(\tau)$ by

$$
\begin{aligned}
j & =\frac{1}{q}\left(1+744 q+196884 q^{2}+21493760 q^{3}+\cdots\right), \\
j^{2} & =\frac{1}{q^{2}}\left(1+1488 q+947304 q^{2}+335950912 q^{3}+\cdots\right), \ldots
\end{aligned}
$$

In particular, by (3.4) and (3.5), we always have

$$
C_{0}^{2}(\tau)= \pm 1
$$

The following theorem is due to a numerical computation. 
TheORem 5. $\lambda_{3}$ satisfies the monic equation

$$
\begin{aligned}
\lambda_{3}^{12} & -4\left(\zeta_{3}+2\right) \lambda_{3}^{11}+22\left(\zeta_{3}+1\right) \lambda_{3}^{10}+\frac{1}{3^{5}}\left(2 \zeta_{3}+1\right)(j-6588) \lambda_{3}^{9} \\
& -\frac{1}{3^{3}} \zeta_{3}(j-2133) \lambda_{3}^{8}+\frac{4}{3^{4}}\left(\zeta_{3}-1\right)(j-1242) \lambda_{3}^{7}+\frac{1}{3^{2}}(j-1044) \lambda_{3}^{6} \\
& +\frac{4}{3^{4}}\left(\zeta_{3}^{2}-1\right)(j-1242) \lambda_{3}^{5}-\frac{1}{3^{3}} \zeta_{3}^{2}(j-2133) \lambda_{3}^{4} \\
& +\frac{1}{3^{5}}\left(2 \zeta_{3}^{2}+1\right)(j-6588) \lambda_{3}^{3}+22\left(\zeta_{3}^{2}+1\right) \lambda_{3}^{2}-4\left(\zeta_{3}^{2}+2\right) \lambda_{3}+1=0 .
\end{aligned}
$$

Until the end of this section, let $\tau(\in \mathfrak{H})$ be again in an imaginary quadratic number field $k(\neq \mathbb{Q}(\sqrt{-1}), \mathbb{Q}(\sqrt{-3}))$, and let $\Omega_{\tau}=[\tau, 1]$ be an $\mathfrak{o}_{k}$-ideal. Then from the above considerations, we see that the value $\lambda_{p}(\tau)$ is a unit outside the prime divisors of $p$. We conjecture that $\lambda_{p}(\tau)$ is a unit if and only if $p$ remains prime in $k$.

Here we consider the case where 3 remains prime in $k$. From Theorem 4 , $\Lambda_{3}(\tau)$ is a unit. Hence the equation in (ii) of Proposition 2 shows that

$$
j^{2}-1512 j+177876 \equiv 0\left(\bmod 3^{9}\right) \quad \text { and } \quad 8 j+2214 \equiv 0\left(\bmod 3^{4}\right) .
$$

This means that $j=3^{3}+3^{4} \theta$ with an integer $\theta$ in $\mathcal{H}_{k}$, the Hilbert class field of $k$, such that $\theta^{2} \equiv 0(\bmod 3)$. Hence the coefficients of the equation in Theorem 5 are all in $\mathbb{Z}\left[\zeta_{3}, j\right]$. Thus we have the following

THEOREM 6. Under the above notations, $\lambda_{3}(\tau)$ is a unit if and only if 3 remains prime in $k$.

Appendix. In the proof of Theorem 1 and in the computation for the numerical example (Theorem 5), we used the following expansion formula for the Weierstrass $\wp$-function.

Proposition A-1 (cf. [9, Ch. 4]). Let $\Omega=[\tau, 1]$ with $\tau$ in $\mathfrak{H}$. Then for $z \in \mathbb{C}$ we have

$$
\frac{1}{(2 \pi i)^{2}} \wp_{\Omega}(z)=\frac{1}{12}+\sum_{m \in \mathbb{Z}} \frac{q^{m} q_{z}}{\left(1-q^{m} q_{z}\right)^{2}}-2 \sum_{n=1}^{\infty} \frac{n q^{n}}{1-q^{n}},
$$

where $q=e^{2 \pi i \tau}$ and $q_{z}=e^{2 \pi i z}$.

Let $p$ be an odd prime number. We apply Proposition A-1 for $z$ which represents a non-zero $p$-division point of $\mathbb{C} /[\tau, 1]$. We may write $z=(a \tau+b) / p$ where $0 \leq a, b \leq p-1$ and $(a, b) \neq(0,0)$. From Proposition A-1, it is easy to deduce that

$$
\frac{1}{(2 \pi i)^{2}} \wp \Omega_{\tau}\left(\frac{a \tau+b}{p}\right)-\frac{1}{12}=R\left(q^{1 / p}\right),
$$


where $q^{1 / p}=e^{2 \pi \tau / p}$ and $R(X)$ is a power series in $X$ whose coefficients belong to $\mathbb{Z}\left[\zeta_{p}\right]$ with $\zeta_{p}=e^{2 \pi i / p}$. In order to determine the leading term of the $q$-expansion of $\Lambda_{p}(\tau)$ at $\infty$, we used the following

Lemma A-2 (cf. [3, Ch. 8]). Under the above notations,

$$
\frac{1}{(2 \pi i)^{2}} \wp_{\Omega_{\tau}}\left(\frac{a \tau+b}{p}\right)-\frac{1}{12}
$$

has q-expansion at $\infty$ in $\mathbb{Z}\left[\zeta_{p}\right]\left[\left[q^{1 / p}\right]\right]$ with leading term

$$
\begin{cases}\zeta_{p}^{b} /\left(1-\zeta_{p}^{b}\right)^{2} & \text { if } a=0, \\ \zeta_{p}^{b} q^{a / p} & \text { if } 0<a<p / 2, \\ \zeta_{p}^{-b} q^{(p-a) / p} & \text { if } p / 2<a<p .\end{cases}
$$

\section{References}

[1] T. M. Apostol, Modular Functions and Dirichlet Series in Number Theory, Grad. Texts in Math. 41, Springer, 1976.

[2] A. Borel, S. Chowla, C. S. Herz, K. Iwasawa and J.-P. Serre, Seminar on Complex Multiplication, Lecture Notes in Math. 21, Springer, 1966.

[3] Ph. Cassou-Noguès and M. J. Taylor, Elliptic Functions and Ring of Integers, Progr. Math. 66, Birkhäuser, Basel, 1987.

[4] J. Cougnard, Modèle de Legendre d'une courbe elliptique à multiplication complexe et monogénéité d'anneaux d'entiers, Acta Arith. 54 (1990), 191-212.

[5] J. Cougnard et V. Fleckinger, Modèle de Legendre d'une courbe elliptique à multiplication complexe et monogénéité d'anneaux d'entiers II, ibid. 55 (1990), 75-81.

[6] M. Deuring, Die Klassenkörper der komplexen Multiplikation, Enzykl. d. Math. Wiss., Bd. 1/2, Heft 10, Teil II, Stuttgart, 1958.

[7] N. Koblitz, Introduction to Elliptic Curves and Modular Forms, 2nd ed., Grad. Texts in Math. 97, Springer, 1993.

[8] D. Kubert and S. Lang, Modular Units, Grundlehren Math. Wiss. 244, Springer, 1973.

[9] S. Lang, Elliptic Functions, Addison-Wesley, 1973.

[10] R. Schertz, Zur Theorie der Ringklassenkörper über imaginär quadratischen Zahlkörpern, J. Number Theory 10 (1978), 70-82.

Department of Mathematics

Kyushu-Tokai University

9-1-1 Toroku

Kumamoto 862-8652, Japan

E-mail: hhayashi@ktmail.ktokai-u.ac.jp

Received on 3.8.2005

and in revised form on 25.7.2006 